\title{
CIRCADIAN AND SEASONAL RHYTHMS
}

\section{The biological clock tunes the organs of the body: timing by hormones and the autonomic nervous system}

\author{
R M Buijs, C G van Eden, V D Goncharuk and A Kalsbeek \\ Netherlands Institute for Brain Research, Meibergdreef 33, 1105 AZ Amsterdam, The Netherlands \\ (Requests for offprints should be addressed to R M Buijs; Email: r.buijs@nih.knaw.nl)
}

\begin{abstract}
The biological clock, the suprachiasmatic nucleus $(\mathrm{SCN})$, is essential for our daily well-being. It prepares us for the upcoming period of activity by an anticipatory rise in heart rate, glucose and cortisol. At the same time the 'hormone of the darkness', melatonin, decreases. Thus, the time-ofday message penetrates into all tissues, interestingly not only by means of hormones but also by a direct neuronal influence of the SCN on the organs of the body. The axis between the SCN and the paraventricular nucleus of the hypothalamus (PVN) is crucial for the organization/ synchronization of the neuroendocrine and autonomic nervous system with the time of day. This SCNneuroendocrine PVN axis takes care of a timely hormonal
\end{abstract}

secretion. At the same time, the SCN-autonomic PVN axis fine-tunes the organs by means of the autonomic nervous system for the reception of these hormones. Finally, the similar organization of the projections of the human SCN as compared with that in the rodent brain suggests that these basic principles of neuroendocrine autonomic interaction may also be true in the human. The physiological data collected in humans thus far seem to support this hypothesis, while pathological changes in the SCN of humans suffering from depression or hypertension indicate a role for the SCN in the etiology of these diseases. Journal of Endocrinology (2003) 177, 17-26

\section{Cellular clocks and the suprachiasmatic nucleus (SCN)}

All life on earth depends on the presence of the sun, which, due to the endless rotation of the earth around its own axis, exposes all organisms on its surface to the daily change in light intensity. From the simplest algae to mammals, nearly all organisms use sunlight to adjust their period of activity to optimize survival. Consequently, organisms have adapted their life style in such a way that they organize their activities in 24-h cycles determined by sunrise and sunset. For this purpose, cellular clock mechanisms have evolved that are sensitive to light and allow anticipation of the period of activity. For this anticipation, most organisms make use of the sunlight that penetrates deeply into tissues and cells. The significance of this observation is that consequently most, if not all, cells of organisms have a (biochemical) system coupled with a molecular mechanism, to drive a cellular clock (Hsu et al. 1996, Plautz et al. 1997, Jin et al. 1999, Van der Horst et al. 1999, Lee et al. 2000, Delauney et al. 2001, Whitmore et al. 2001). This evolutionary pressure has been so strong that even in fish, such as the zebrafish, this property has been retained such that light is directly able to affect clock mechanisms in organs or muscle tissue (Delaunay et al. 2001). Proteins such as the cryptochromes, which are conserved from plants to humans (Hsu et al. 1996), have been shown to play a very important role in this light-transducing pathway. In evolution, cryptochromes were first essential for light reception in plants, later their function shifted to the molecular clockwork mechanism in mammals where cryptochromes are essential components of the circadian clock (Van der Horst et al. 1999). From the lowest invertebrate species to mammals, a centralization of the light-receptive elements has taken place. Together with the increasing complexity of the nervous system, the tendency can be observed of the movement and/or concentration of circadian clockcontaining cells to the light-transducing parts of the nervous system. Clock mechanisms have evolved from algae and fungi, which are organisms that are exposed to sunlight but that have, of course, no neurons (Lee et al. 2000 ) to fruit flies and zebrafish with independent clockcontaining cells in all parts of the body, but with a high concentration in the retina (Plautz et al. 1997); interestingly, in these latter organisms light is able to penetrate all 
tissues. In contrast, in birds light-receptive cells are present in retina and pineal (Menaker 1971) while in mammals the light signal can only reach the central nervous system (CNS) via the retina and by glutamate secretion from retinal terminals (Ding et al. 1997). In mammals, independent clock cells are, as yet, only shown in the retina itself and in the SCN, the latter located immediately on top of the optic nerve. In this location, the SCN receives the light information from the retina. In spite of the fact that cells of many organs have the ability to retain a rhythmic function and express clock genes with a circadian rhythmicity for a few days, this property disappears without daily enforcement, i.e. without the SCN (Yamazaki et al. 2000). In the present paper, we shall present the view that the SCN transmits its daily message to the rest of the body by means of hormonal rhythms and multisynaptic autonomic connections onto the organs of the body. Finally, this synchronized output of the SCN results in the circadian expression of clock proteins in the tissues of the organs of the body with the associated metabolism (Yamazaki et al. 2000). When examining which SCN outputs might be responsible for this synchronization, a series of recent studies has demonstrated that the peripheral clock can be driven by a number of different entraining factors, such as food intake (Damiola et al. 2001), hormones (Balsalobre et al. 2000), and temperature/energy balance (Brown et al. 2002).

Since it is the fundamental property of independent clock cells (SCN cells in mammals) that they have an activity cycle of approximately $24 \mathrm{~h}$ (circadian), much effort has been directed towards research aimed at unraveling the cellular mechanisms behind this property of the clock. In particular, the molecular mechanisms behind this rhythm have mesmerized and challenged molecular biologists. Today, many components of this molecular machinery have been exposed; indicating that the molecular component of the clock consists of gene-protein-gene feedback loops whereby the protein has a negative feedback on its own transcription and stimulates the transcription of other clock proteins (Hsu et al. 1996, Plautz et al. 1997, Jin et al. 1999, Van der Horst et al. 1999, Lee et al. 2000, Delauney et al. 2001, Whitmore et al. 2001). However, no data are as yet available to explain how this molecular loop is linked to the cellular mechanism that controls the circadian electrical activity-inactivity cycle of the neuron. In the present review, we concentrate on demonstrating how these clock cells in mammals with their complex CNS transmit their signal to the rest of the organism. Attention will be focused on the question as to how the message of the sun is translated into a daily pattern of SCN neuronal activity and, more importantly, transmitted to all those parts of the body of mammals that the sunlight cannot penetrate anymore but where clock-like mechanisms still follow the signal of the main oscillator (Yamazaki et al. 2000, Brown et al. 2002).

\section{The neuronal targets of the clock}

Precise injections of anterograde tracers into the SCN uncovered the neuronal targets of the clock (Watts et al. 1987). Up to now two important aspects of SCN targets have been revealed: (1) targets are mainly restricted to the midline regions of the hypothalamus with only two extra hypothalamic targets, the lateral geniculate nucleus (LGN) and the paraventricular nucleus of the thalamus (PVT) and (2) the hypothalamic targets of the SCN consist of cell groups that are known for their influence on the autonomic and the endocrine systems.

Since SCN transplantation is able to restore locomotor activity patterns in SCN-lesioned animals (Ralph et al. 1990), and these SCN transplants most of the time lack outgrowth of fibers, the question arose as to whether the output of the SCN indeed needs to be directed to specific targets in the CNS or whether diffusion of its transmitters might be sufficient to transfer its message. The first experiments conducted to solve this issue demonstrated that restoration of circadian activity could even be achieved by placing an SCN transplant encapsulated within a semi-permeable membrane into the cerebral ventricle (Silver et al. 1996). These observations suggest that the SCN message can (at least partly) be conveyed by means of diffusible substances. This idea was reinforced by recent observations on new signaling molecules of the SCN that may be transmitted by diffusion (Kramer et al. 2001, Cheng et al. 2002). However, the neurotransmitter $(\gamma$-amino butyric acid; GABA) and glutamate, for which, in contrast to peptides, high-affinity uptake sites are present, also play a role in the synaptic transmission of SCN output (Hermes et al. 1996, Csaki et al. 2000, Cui et al. 2002). Therefore, it is highly questionable whether all SCN functions can be restored adequately by transplantation without full regrowth of the connections. This doubt was substantiated by experiments in which SCN transplantation resulted in restoration of circadian locomotor activity in SCN-lesioned animals, whereas the 24-h rhythm in cortisol secretion or the seasonal rhythm in gonadal function could not be restored (Meyer-Bernstein et al. 1999). Consequently, the exact connection to target neurons and a more precisely tuned message at the synaptic cleft will be essential for a full transmission of the signal of the clock. These observations warrant a more accurate analysis of targets and putative transmitters of the SCN, combined with functional markers in the target areas. Several studies have shown that the SCN specifically targets the following: (1) gonadotropin-releasing hormone $(\mathrm{GnRH})$ neurons in the rostral forebrain (Van der Beek et al. 1997) and neurons that contain estrogen nuclear receptors (Delaiglesia et al. 1995), providing the anatomical basis for the influence of the SCN on the reproductive cycle in females and on the daily testosterone surges in males; (2) neurons that are activated during stress mainly around the paraventricular nucleus of the hypothalamus 
(PVN) (Buijs et al. 1993b), providing the anatomical basis that explains the role of the SCN in stress-controlled corticosteroid regulation (Kalsbeek et al. 1996b, Choi et al. 1998); (3) dopamine-containing neurons probably implicated in the regulation of prolactin secretion by dopamine release in the tubular infundibular region (Horvath 1997); (4) neurons projecting to the autonomic nervous system (parasympathetic and sympathetic), providing the anatomical basis for the circadian control of the pineal, adrenal, pancreas, liver, ovaries and other organs (Larsen et al. 1998, Gerendai et al. 2000, Buijs et al. 2001); (5) neurons below the PVN (subPVN) and within the dorsomedial nucleus of the hypothalamus $(\mathrm{DMH})$ seem essential for the transmission of sleep/wake information (Aston-Jones et al. 2001); (6) magnocellular neurons, having a putative role in the secretion of neurohypophysial hormones (Hermes et al. 1996, 2000).

As a result of these observations, we propose that the SCN utilizes at least four different types of neuronal targets to pass on its circadian signal: (1) direct communication with neuroendocrine neurons; (2) direct communication with autonomic (PVN) neurons to communicate the timeof-day signal to the different organs of the body; (3) communication with hypothalamic structures in between the SCN and PVN, such as the subPVN, DMH and medial preoptic area (MPA), which may serve as an intermediate target between SCN and neuroendocrine and autonomic neurons; (4) communication to areas outside the hypothalamus, such as the LGN and PVT in order to synchronize hypothalamic-induced behavior and locomotor activity. Of course, we still have to explain how these hypothalamic structures like the PVN are able to organize the motor behavior associated with SCN functioning. Since no direct PVN projections are present to CNS structures that may co-ordinate motor behavior, we would propose that its projections to the nucleus tractus solitarius (NTS) are used for this purpose. According to this view, the NTS may then signal the CNS information integrated with the actual information on the state of the internal organs to cortical structures in order to initiate the appropriate motor behavior.

\section{The organization of the SCN}

The SCN cannot be viewed as a homogeneous structure but is composed of different neuronal elements, each probably with its own specific function. The neurons which are the components of these elements have intensive interconnections and interaction, as was demonstrated by ultrastructural and immunochemical studies (Buijs et al. 1995, Romijn et al. 1997). Within the SCN at least a certain specialization should exist. The SCN consists of different neurons containing different neuropeptides, such as vasopressin (VP), vasoactive intestinal peptide (VIP), gastrin-releasing peptide or somatostatin. It has been demonstrated that several of these neurons co-localize GABA or glutamate. Ultrastructural studies have revealed that about $30 \%$ of the SCN terminals contain GABA (Buijs et al. 1995, Castel \& Morris 2000), while electrophysiological studies showed the presence of glutamate as an additional SCN transmitter (Hermes et al. 1996, Cui et al. 2002). The combination of a large variety of peptides with or without amino acid transmitters within a single nucleus endows the SCN with a rich variety of signaling properties. This set of SCN neurons with their respective neurotransmitters serves to convey the circadian and light/dark signal to hypothalamic target structures (Kalsbeek et al. 1996b, 2000, Buijs \& Kalsbeek 2001).

\section{Circadian control of hormone secretion}

The circadian peak of blood corticosterone is controlled by the SCN but the signal is generated in the PVN where corticotropin-releasing hormone (CRH)containing neurons regulate the secretion of adrenocorticotropin (ACTH) from the pituitary. Since VP is a peptidergic neurotransmitter of the SCN with terminals within and around the PVN, and VP levels show a circadian variation with a high secretion during the daytime period, this peptide was a logical choice to investigate its putative value for the regulation of corticosterone secretion at the level of the PVN.

Infusion of VP in intact and SCN-lesioned animals resulted in an almost immediate inhibition of corticosterone and, to a lesser extent, ACTH secretion. In contrast, a VP antagonist was very effective in increasing corticosterone secretion, but only in intact animals, whereas it was ineffective in SCN-lesioned animals, demonstrating the endogenous role of SCN-derived VP in the PVN of intact animals (Kalsbeek et al. 1996b). The most effective site of infusion, however, proved to be the $\mathrm{DMH}$, an area heavily innervated by VP fibers originating from the SCN. These observations and further anatomical and electrophysiological evidence indicate that the circadian signal reaches the CRH neurons mainly in an indirect manner. The DMH is known to contain GABAergic neurons that project to the medial parvocellular part of the PVN (Roland \& Sawchenko 1993). Later electrophysiological studies support the hypothesis that VP may excite these GABA-ergic interneurons, resulting in an inhibition of neuroendocrine neurons in the PVN (Hermes et al. 2000) (Fig. 1). SCN projections may also reach PVN neuroendocrine neurons directly via PVN dendrites protruding towards the third ventricle, an area that is, in addition to the part ventral to the PVN, rich in SCN-derived fibers (Buijs et al. 1993b).

It has been demonstrated that VP originating in the SCN and targeted to the DMH plays an important inhibitory role in ACTH and corticosterone secretion. By infusing VP antagonists over the 24-h period, a peak of 


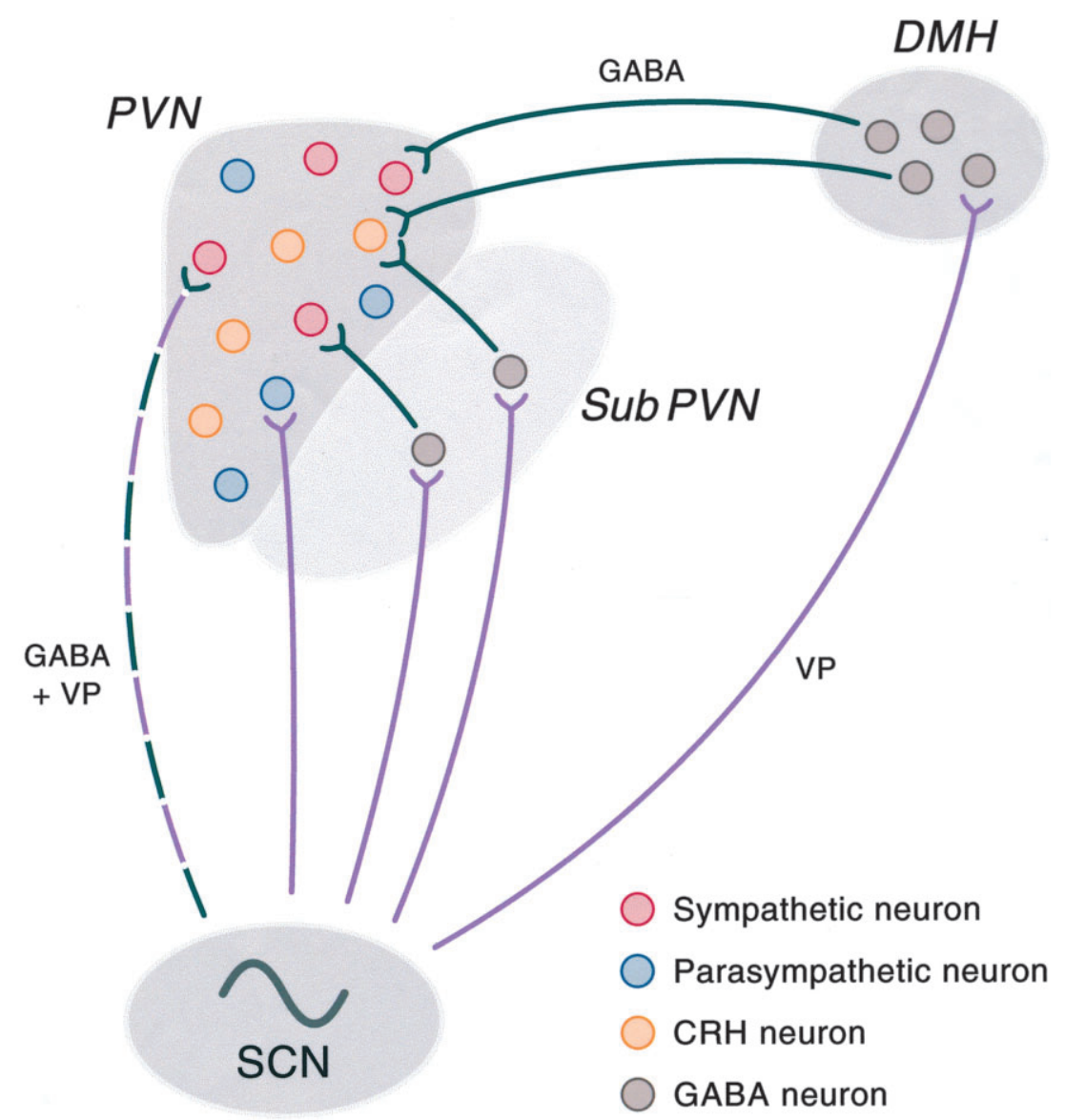

Figure 1 This provides a detailed anatomical scheme of demonstrated and putative connections of the SCN in the rat brain that is used by the biological clock to enforce its rhythm onto the neuroendocrine and autonomically projecting neurons of the PVN. Three major transmitters of the SCN are indicated here: GABA, glutamate and VP. VP, which is released during the daytime (Gillette \& Reppert 1987) is proposed to inhibit CRH neurons via GABA-ergic interneurons in the subPVN and DMH. At the same (day) time, it is proposed that the activity of the sympathetically projecting neurons in the PVN (e.g. projecting to the pineal) is suppressed, by means of a GABA-ergic mechanism, resulting in, for example, a decrease of melatonin secretion. It is proposed that parasympathetically projecting neurons will not receive a direct GABA-ergic input from the SCN but rather a stimulatory VP or VP+glutamate-activating input. During the dark period, when the animal is active, this network should lead to an activation of the sympathetically projecting neurons of the PVN and to an inhibition of the parasympathetically projecting ones. The opposite is proposed to be true for the daytime period, when the animal is inactive.

corticosterone was revealed which encompasses a longer period than the usual corticosterone curve (Kalsbeek et al. 1996b). This observation suggests that the corticosterone curve is organized by at least two mechanisms from the SCN: a stimulating component of an as yet undetermined nature and an inhibiting component, and the daily VP secretion from SCN terminals within the DMH (Fig. 2). Evidently the purpose of these mechanisms is not only to drive the daily corticosterone peak in mammals but also to maintain, for example, stress-induced corticosterone excursions within the physiological range appropriate for the stress and appropriate for that moment of the day/night cycle (Buijs et al. 1993a). Furthermore, a number of independent anatomical and physiological studies suggest that the SCN controls other hormones in a similar fashion. For example, SCN fibers contact estrogen-concentrating neurons in the MPA (Delaiglesia et al. 1995), whereas the direct contact with GnRH neurons remains limited (Delaiglesia et al. 1995, Van der Beek et al. 1997). However, also in the MPA, VP secretion from the SCN serves to shape the GnRH peak in female rats (Palm et al. 1999). It seems logical that the stimulatory role of VP for the $\mathrm{GnRH}$ axis coincides with an inhibitory role of SCN-derived VP on corticosterone secretion. The SCN 

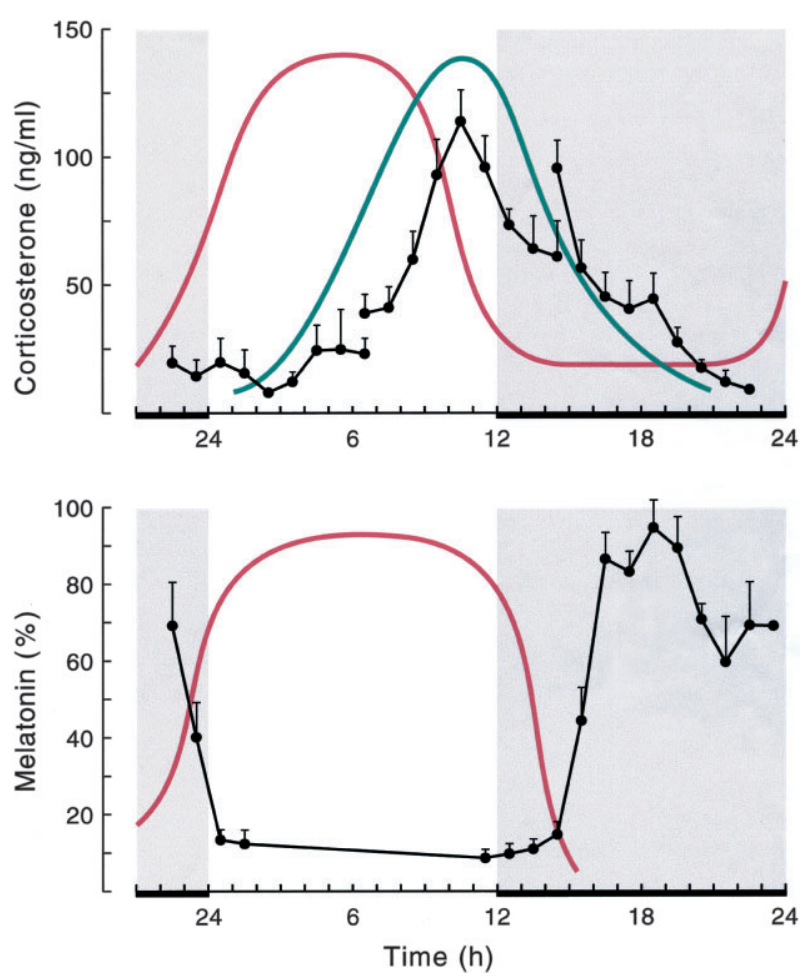

Figure 2 This represents the daily peak in plasma corticosterone and melatonin as observed in intact male rats (solid horizontal bars and shaded areas represent night-time). (Upper figure) Spaced infusions of VP antagonist over the $24 \mathrm{~h}$ clearly revealed a stimulatory peak of corticosterone for which an as yet unknown $\mathrm{SCN}$ transmitter (green) is responsible. In addition, it revealed that a strong endogenous inhibitory action of VP (red line in upper figure) is the strongest in the middle of the inactive period. The stimulatory action on corticosterone secretion is indicated by the green curve. (Lower figure) Infusions of GABA agonists inhibited melatonin secretion at night and the GABA antagonists bicucullin infusion into the PVN resulted in a daytime secretion of melatonin. Consequently, we propose that the secretion of GABA from SCN terminations in the PVN (red line in lower figure) shapes the nightly melatonin peak. The three colored lines indicate at least three temporal separated actions of individual SCN neurons.

reduces the stress response on the one hand, while stimulating sexual receptivity on the other.

Examining the way the SCN affects melatonin secretion offers an additional view of the possibilities of the SCN to control hormone secretion. Hereto, the SCN reaches the autonomic neurons of the PVN that project to the intermediolateral column of the spinal cord (IML) where preganglionic sympathetic neurons are located that, for example, control the outflow to the pineal (Larsen et al. 1998, Teclemariam Mesbah et al. 1997, 1999). Muscimol, a specific GABA agonist, infused into the PVN was able to decrease night-time melatonin secretion, indicating that inhibition of PVN neurons that organize the melatonin peak prevent the appearance of the peak (Kalsbeek et al. 1996a). Since light activates SCN neurons by means of retinohypothalamic glutamate secretion and at the same time results in the immediate inhibition of melatonin at night, it was hypothesized that activation of SCN neurons would cause a release of GABA from their terminals in the PVN and consequently an inhibition of melatonin secretion. In order to test this hypothesis, it was investigated to what extent GABA, under physiological circumstances, would be responsible for the melatonin inhibition by light. Interestingly, the infusion of the GABA antagonist bicucullin bilaterally into the PVN completely prevented the light-induced inhibition of melatonin and even resulted in a melatonin peak during the daytime period (Kalsbeek et al. 2000). It was concluded that the SCN regulates melatonin secretion, to a large part, by a GABA-mediated inhibition of autonomic PVN neurons. Recently, the SCN GABA-ergic control of autonomic PVN neurons was supported by the results of Cui et al. (2002) who demonstrated that identified spinal cord projecting neurons in the PVN were inhibited by GABA after SCN stimulation. Consequently, these autonomic PVN neurons should be active during the night, which should result in melatonin secretion. This conclusion is corroborated by observations that lesioning of the PVN results in low night-time melatonin levels, while SCN lesions result in high melatonin levels around the clock (Kalsbeek et al. 2000). It will be extremely interesting to investigate whether GABA alone, by a daily inhibition of PVN neurons, is responsible for the melatonin peak, or whether the activity of the PVN neurons is also SCN induced. The fact that in SCN-X animals melatonin is high but just half the normal night levels supports this idea. Consequently, glutamate, which also plays a role in SCN neurotransmission to the autonomous PVN, could be responsible for this increase.

The picture emerges of both GABA and VP playing a role in transmitting the daytime message of the SCN. Herein, it is interesting that VP is excitatory to most neurons and GABA inhibitory. Since GABA is colocalized in $30 \%$ of the VP neurons, it would be of great interest to examine the precise targets of VP terminals that do and do not co-localize GABA. Since SCN terminals originating from VP neurons reach both parasympathetic and sympathetic projecting neurons in the PVN, it seems possible that in these VP connections the addition of GABA in part of them allows the SCN to differentiate between parasympathetic and sympathetic output. The SCN-mediated GABA-ergic inhibition on melatonin secretion by day suggests, at least, that the activation of sympathetic neurons of the PVN is suppressed by an SCN-GABA neurotransmission (Figs 1 and 2).

\section{The three-way approach of the SCN: behavior and synchronized autonomic and hormonal output}

The fact that the SCN is crucial for the organization of the melatonin surge during the night indicates that autonomic 


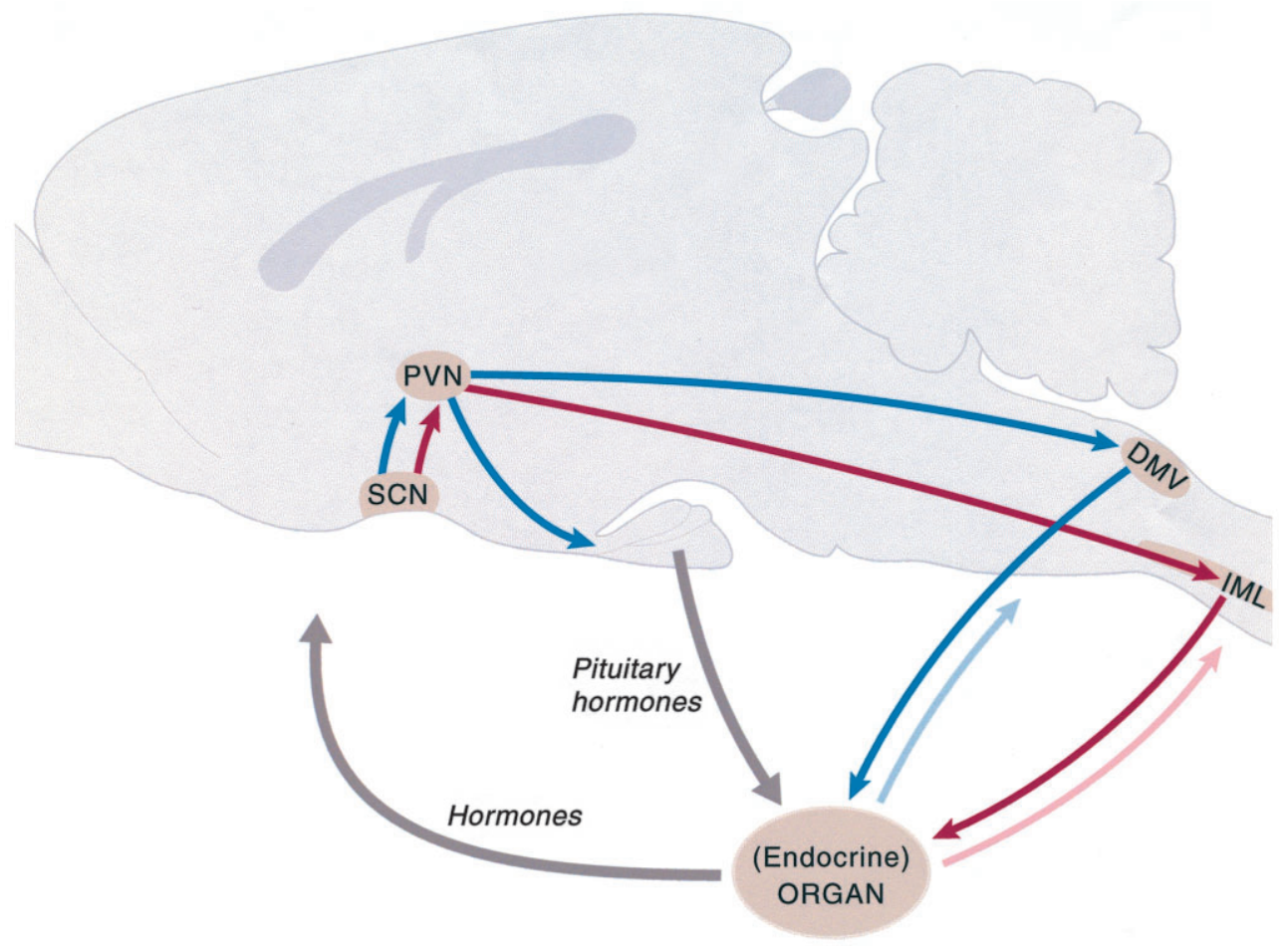

Figure 3 This illustrates the main pathways by which the SCN communicates its circadian signal to the body. Via its connections with the PVN, three different output systems are affected: (1) the neuroendocrine neurons of the PVN responsible for the control of the pituitary hormones, (2) parasympathetically projecting neurons in the PVN that specifically target the dorsal motor nucleus of the vagus (DMV) and (3) sympathetically projecting neurons in the PVN that specifically target the spinal cord preganglionic neurons located in the IML. The peripheral organs are reached via these three mechanisms. The neuronal message, in fact, prepares and sets the sensitivity of the organs for the arrival of the hormones. In return, the neuronal and hormonal message of the organs will feed back to the CNS and SCN as indicated by the three arrows directed to the brain.

control is at least one important aspect of SCN functioning. This idea is supported by early data suggesting that, in addition to controlling the pineal, the SCN may also have an important neuronal influence on the adrenal cortex. In fact, evidence of a circadian control of the adrenal sensitivity for ACTH was reported over 20 years ago (Kaneko et al. 1980). Transneuronal tracing and physiological experiments finally provided proof that, apart from the classical neuroendocrine control of the adrenal cortex by the PVN-CRH-ACTH-adrenal cortex cascade, the autonomic projections of the SCN via the PVN to the IML determines the daily changes in sensitivity of the adrenal to ACTH (Kaneko et al. 1980, Kalsbeek et al. 1996b, Buijs et al. 1999). These experiments demonstrated that the SCN utilizes a dual mechanism to organize an optimum secretion of corticosterone (1) via VP and as yet unknown other transmitters, control of the neuroendocrine HPA axis and (2) via VP and possibly GABA, autonomic control of the adrenal (Figs 2 and 3). Finally, it should be noted that in the DMH, VP as a neurotrans- mitter of the SCN opposes the hormonal role of VP when it is released from PVN terminals in the median eminence. As a neurotransmitter in the DMH, VP inhibits corticosterone secretion and as a hormone in the portal circulation it stimulates it.

We would like to generalize this observation and propose that the SCN affects hormone secretion and affects the sensitivity of the target organs of these hormones by neuronal mechanisms (Fig. 1). For example, anatomical and physiological evidence shows that the level of insulin secretion from the pancreas is influenced by the SCN (Nagai et al. 1996, Kalsbeek \& Strubbe 1998, La Fleur et al. 2001). In addition, when the SCN stimulates glucose secretion from the liver, at the same time it stimulates uptake of glucose by other tissues (La Fleur et al. 2001). This timely orchestrated action of the SCN on glucose metabolism prepares our body just before waking for the coming period of activity. Injection of transneuronal tracers into various organs and tissues ranging from heart to ovaries and from white to brown adipose tissue results in 
the labeling of neurons in the SCN via the sympathetic and parasympathetic branches of the autonomic nervous system (Gerendai et al. 2000, La Fleur et al. 2000, Buijs et al. 2001, Kreier et al. 2002). These data demonstrate that the SCN may transmit its circadian message, influencing the activity/rest cycle of peripheral organs, not only by means of the secretion of hormones that may freely pass all kinds of tissue barriers, such as corticosterone and melatonin, gonadal hormones and thyroid hormones, but also by a direct nervous control of these organs (Fig. 3). Interestingly, SCN neurons are labeled both via the sympathetic and parasympathetic system, indicating that the SCN may indeed support both the activity and the rest period of the circadian cycle (Figs 1 and 3).

\section{The human SCN}

Most data on SCN anatomy and SCN projections are collected in rodents. Yet it is of the utmost importance to examine whether similar projections and functional connections also hold for the human brain. Several peptide transmitters of the SCN were demonstrated in the human hypothalamus, revealing the presumed axonal projections of SCN neurons by means of staining for VIP and VP. To corroborate the observed staining patterns with the true projections from the SCN, a postmortem tracing technique was developed that allowed the uptake of the anterograde tracer biocytin and its transport in the human postmortem brain. The subsequently revealed hypothalamic projections of the human SCN turned out to be identical to those observed in the rat brain (Dai et al. 1998b). Since the human SCN also targets the DMH, it was also investigated whether the human DMH has the same connections as that of the rat. Also, this study proved that the organization of the projections of this part of the human hypothalamus is comparable with that of the rat; the human DMH has extensive projections to neuroendocrine neurons of the PVN and to the ventral medial hypothalamus (Dai et al. 1998a).

Increased electrical activity of the SCN during daytime as compared with the dark period seems to be a general phenomenon among mammals. Compared with the rat, the secretion of corticosterone and the regulation of activity, heart rate and temperature in humans take place within a completely different moment within the light/ dark cycle. The electrical activity of the SCN, the signal of the SCN, however, is the same as the signal of the SCN to the human PVN and DMH. Therefore, with respect to the control of these functions, they should be translated in an opposite way to that of the nocturnal rat.

The observations in rodents and humans indicate an important role for the SCN in the regulation of bodily functions through the control of hormone secretion on the one hand and via the autonomic nervous system on the other. Evidence is accumulating that SCN functioning is
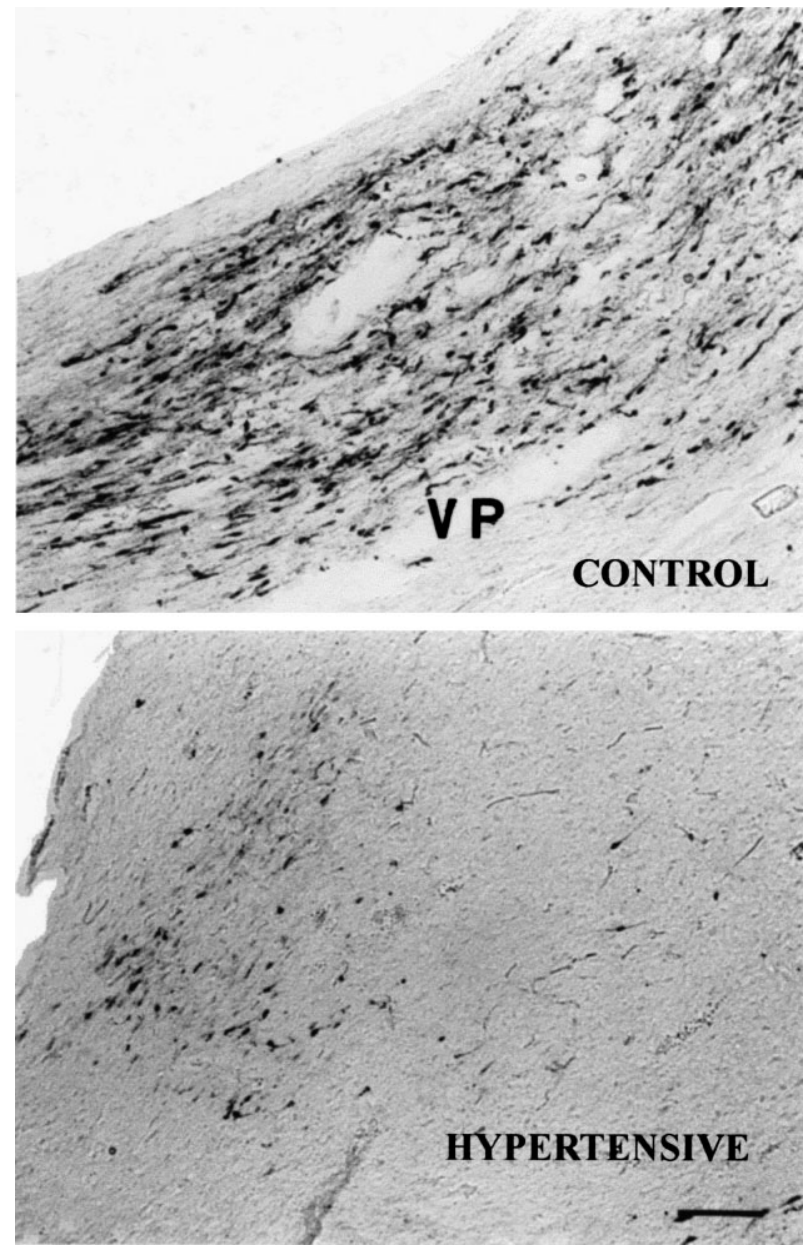

Figure 4 This illustrates the postmortem staining for VP in the $\mathrm{SCN}$ of a control person and of a hypertensive person. Both sections were chosen because they represent the mid-portion of the SCN with the highest number of neurons stained for VP. The figure demonstrates a clear reduction in immunoreactivity in VP neurons in the human SCN in patients who had a long history of hypertension. This dramatic decrease in VP staining, coinciding with a similar decrease in neurotensin or VIP staining, indicates that either these changes are the result of hypertensive feedback or one of the causes of hypertension. (Bar=100 $\mu \mathrm{m}$ ). (Reprinted with permission from Goncharuk et al. 2001.)

affected in several chronic diseases, such as hypertension, diabetes and depression. For a long time now physiological studies have indicated disturbances in the circadian rhythm in patients suffering from these diseases (Pickering 1990, Yamamoto et al. 1999). Recently, we demonstrated the anatomical basis for these disturbances in hypertensive patients: a severe decrease in staining for several SCN neurotransmitters (Goncharuk et al. 2001) (Fig. 4). Interestingly, the finding that followed this observation was that in the same patients an enhanced activity of PVN CRH neurons was observed, which would suggest that, in the human, the SCN would also have an inhibitory role on the 
$\mathrm{CRH}$ neurons in the PVN. This intriguing observation either suggests that SCN activity is changed by a different autonomic feedback in hypertension or that SCN changes precede the development of hypertension. Since the anatomy of the SCN is changed in spontaneous hypertensive rats (SHR) and transplantation of the hypothalamus containing the SCN from SHR to normotensive rats induces hypertension (Goncharuk et al. 2002), the suggestion is strong that a changed SCN may precede the development of hypertension. Furthermore, evidence of the last few years has provided support for the notion that circadian disturbances can already be detected before the development of diabetes or hypertension (Borghi et al. 1986, Eilam et al. 1991). A less active SCN may prepare an individual less efficiently for the new period of activity. Everyday repetition of this strain for many years may slowly result in the development of hypertension. The fact that cardiovascular accidents precipitate during the morning hours at the onset of the period of activity only supports this hypothesis.

In conclusion, the present evidence suggests that the SCN utilizes three major mechanisms to synchronize the physiology of the organism to the daily change in activity. These mechanisms activate or inhibit (1) hypothalamic centers that facilitate certain behavior that can be associated with activity or inactivity, (2) neuroendocrine hypothalamic centers that are responsible for hormone secretion and (3) preautonomic hypothalamic neurons that affect parasympathetic and sympathetic autonomic centers in brain stem and spinal cord. This action of the SCN on these target areas is of course not isolated but instead is highly synchronized in order to prepare the whole organism, brain and organs for the coming period of activity. The first postmortem analyses of the SCN in human physiological disorders suggest that indeed the functionality of the biological clock may be affected in diseases such as depression and hypertension (Zhou et al. 2001, Goncharuk et al. 2002).

\section{References}

Aston-Jones G, Chen S, Zhu Y \& Oshinsky ML 2001 A neural circuit for circadian regulation of arousal. Nature Neuroscience $\mathbf{4}$ 732-778

Balsalobre A, Brown SA, Marcacci L, Tronche F, Kellendonk C, Reichardt HM, Schutz G \& Schibler U 2000 Resetting of circadian time in peripheral tissues by glucocorticoid signaling. Science 289 2344-2347.

Borghi C, Costa FV, Boschi S, Mussi A \& Ambrosioni E 1986 Predictors of stable hypertension in young borderline subjects: a five-year follow-up study. Journal of Cardiovascular Pharmacology 8 S138-S141.

Brown S, Zumbrunn G, Fleury-Olela F, Preitner N \& Schibler U 2002 Rhythms of mammalian body temperature can sustain peripheral circadian clocks. Current Biology 12 1574-1583.

Buijs RM \& Kalsbeek A 2001 Hypothalamic integration of central and peripheral clocks. Nature Reviews Neuroscience 2 521-526.

Buijs RM, Kalsbeek A, Van Der Woude TP, Van Heerikhuize JJ \& Shinn S 1993a Suprachiasmatic nucleus lesion increases corticosterone secretion. American Journal of Physiology 264 R1186-R1192.

Buijs RM, Markman M, Nunes-Cardoso B, Hou YX \& Shinn S $1993 b$ Projections of the suprachiasmatic nucleus to stress-related areas in the rat hypothalamus: a light and electronmicroscopic study. Journal of Comparative Neurology 335 42-54.

Buijs RM, Wortel J \& Hou YX 1995 Colocalization of gammaaminobutyric acid with vasopressin, vasoactive intestinal peptide, and somatostatin in the rat suprachiasmatic nucleus. Journal of Comparative Neurology 358 343-352.

Buijs RM, Wortel J, Van Heerikhuize JJ, Feenstra MG, Ter Horst GJ, Romijn HJ \& Kalsbeek A 1999 Anatomical and functional demonstration of a multisynaptic suprachiasmatic nucleus adrenal (cortex) pathway. European Journal of Neuroscience 11 1535-1544.

Buijs RM, Chun SJ, Niijima A, Romijn HJ \& Nagai K 2001 Parasympathetic and sympathetic control of the pancreas: a role for the suprachiasmatic nucleus and other hypothalamic centers that are involved in the regulation of food intake. Journal of Comparative Neurology 431 405-423.

Castel M \& Morris JF 2000 Morphological heterogeneity of the GABAergic network in the suprachiasmatic nucleus, the brain's circadian pacemaker. Journal of Anatomy 196 1-13.

Cheng MY, Bullock CM, Li C, Lee AG, Bermak JC, Belluzzi J, Weaver DR, Leslie FM \& Zhou QY 2002 Prokineticin 2 transmits the behavioural circadian rhythm of the suprachiasmatic nucleus. Nature 417 405-410.

Choi S, Wong LS, Yamat C \& Dallman MF 1998 Hypothalamic ventromedial nuclei amplify circadian rhythms: do they contain a food-entrained endogenous oscillator? Journal of Neuroscience $\mathbf{1 8}$ 3843-3852.

Csaki A, Kocsis K, Halasz B \& Kiss J 2000 Localization of glutamatergic/aspartatergic neurons projecting to the hypothalamic paraventricular nucleus studied by retrograde transport of $\left[{ }^{3} \mathrm{H}\right] \mathrm{D}$-aspartate autoradiography. Neuroscience 101 637-655.

Cui LN, Coderre E \& Renaud LP 2002 Glutamate and GABA mediate suprachiasmatic nucleus inputs to spinal-projecting paraventricular neurons. American Journal of Physiology. Regulatory, Integrative and Comparative Physiology 281 R1283.

Dai JP, Vandervliet J, Swaab DF \& Buijs RM 1998a Postmortem anterograde tracing of intrahypothalamic projections of the human dorsomedial nucleus of the hypothalamus. Journal of Comparative Neurology 401 16-33.

Dai JP, Vandervliet J, Swaab DF \& Buijs RM 19986 Postmortem tracing reveals the organization of hypothalamic projections of the suprachiasmatic nucleus in the human brain. Journal of Comparative Neurology 400 87-102.

Damiola F, Le Minh N, Preitner N, Kornmann B, Fleury-Olela F \& Schibler U 2001 Restricted feeding uncouples circadian oscillators in peripheral tissues from the central pacemaker in the suprachiasmatic nucleus. Genes and Development 14 2950-2961.

Delaiglesia HO, Blaustein JD \& Bittman EL 1995 The suprachiasmatic area in the female hamster projects to neurons containing estrogen receptors and GnRH. Neuroreport 6 1715-1722.

Delaunay F, Thisse C, Marchand O, Laudet V \& Thisse B 2001 An inherited functional circadian clock in zebrafish embryos. Science 289 297-300.

Ding JM, Faiman LE, Hurst WJ, Kuriashkina LR \& Gillette MU 1997 Resetting the biological clock: mediation of nocturnal CREB phosphorylation via light, glutamate, and nitric oxide. Journal of Neuroscience 17 667-675.

Eilam R, Malach R, Bergmann F \& Segal M 1991 Hypertension induced by hypothalamic transplantation from genetically hypertensive to normotensive rats. Journal of Neuroscience $\mathbf{1 1}$ 401-411.

Gerendai I, Toth IE, Boldogkoi Z, Medveczky I \& Halasz B 2000 CNS structures presumably involved in vagal control of ovarian function. Journal of the Autonomic Nervous System 80 40-45. 
Gillette MU \& Reppert SM 1987 The hypothalamic suprachiasmatic nuclei: circadian patterns of vasopressin secretion and neuronal activity in vitro. Brain Research Bulletin 19 135-139.

Goncharuk VD, Van Heerikhuize J, Dai JP, Swaab DF \& Buijs RM 2001 Neuropeptide changes in the suprachiasmatic nucleus in primary hypertension indicate functional impairment of the biological clock. Journal of Comparative Neurology 431 320-330.

Goncharuk VD, Van Heerikhuize J, Swaab DF \& Buijs RM 2002 Paraventricular nucleus of the human hypothalamus in primary hypertension: activation of corticotropin-releasing hormone neurons. Journal of Comparative Neurology 443 321-331.

Hermes MLHJ, Coderre EM, Buijs RM \& Renaud LP 1996 GABA and glutamate mediate rapid neurotransmission from suprachiasmatic nucleus to hypothalamic paraventricular nucleus in rat. Journal of Physiology 496 749-757.

Hermes ML, Ruijter JM, Klop A, Buijs RM \& Renaud LP 2000 Vasopressin increases GABAergic inhibition of rat hypothalamic paraventricular nucleus neurons in vitro. Journal of Neurophysics $\mathbf{8 3}$ 705-711.

Horvath TL 1997 Suprachiasmatic efferents avoid phenestrated capillaries but innervate neuroendocrine cells, including those producing dopamine. Endocrinology 138 1312-1320.

Hsu DS, Zhao X, Zhao S, Kazantsev A, Wang RP, Todo T, Wei YF \& Sancar A 1996 Putative human blue-light photoreceptors hCRY1 and hCRY2 are flavoproteins. Biochemistry 35 13871-13877.

Jin X, Shearman LP, Weaver DR, Zylka MJ, De Vries GJ \& Reppert SM 1999 A molecular mechanism regulating rhythmic output from the suprachiasmatic circadian clock. Cell 96 57-68.

Kalsbeek A \& Strubbe JH 1998 Circadian control of insulin secretion and glucose homeostasis is independent of the temporal distribution of feeding. Physiology and Behaviour 63 553-560.

Kalsbeek A, Drijfhout WJ, Westerink BHC, Vanheerikhuize JJ, Vanderwoude TP, Vandervliet J \& Buijs RM 1996a GABA receptors in the region of the dorsomedial hypothalamus of rats are implicated in the control of melatonin and corticosterone release. Neuroendocrinology 63 69-78.

Kalsbeek A, Vanheerikhuize JJ, Wortel J \& Buijs RM 1996 b A diurnal rhythm of stimulatory input to the hypothalamopituitary-adrenal system as revealed by timed intrahypothalamic administration of the vasopressin $\mathrm{V}-1$ antagonist. Journal of Neuroscience 16 5555-5565.

Kalsbeek A, Garidou ML, Palm IF, Van der Vliet J, Simonneaux V, Pevet P \& Buijs RM 2000 Melatonin sees the light: blocking GABA-ergic transmission in the paraventricular nucleus induces daytime secretion of melatonin. European Journal of Neuroscience 12 3146-3154.

Kaneko M, Hiroshige T, Shinsako J \& Dallman MF 1980 Diurnal changes in amplification of hormone rhythms in the adrenocortical system. American Journal of Physiology 239 R309-R316.

Kramer A, Yang FC, Snodgrass P, Li X, Scammell TE, Davis FC \& Weitz CJ 2001 Regulation of daily locomotor activity and sleep by hypothalamic EGF receptor signaling. Science 294 2511-2515.

Kreier F, Fliers E, Voshol PJ, Van Eden CG, Havekes LM, Kalsbeek A, Van Heijningen CL, Sluiter AA, Mettenleiter TC, Romijn JA, Sauerwein HP \& Buijs RM 2002 Selective parasympathetic innervation of subcutaneous and intra-abdominal fat - functional implications. Journal of Clinical Investigation 110 1243-1250.

La Fleur SE, Kalsbeek A, Wortel J \& Buijs RM 2000 Polysynaptic neural pathways between the hypothalamus, including the suprachiasmatic nucleus, and the liver. Brain Research 871 50-56.

La Fleur SE, Kalsbeek A, Wortel J, Fekkes ML \& Buijs RM 2001 A daily rhythm in glucose tolerance: a role for the suprachiasmatic nucleus. Diabetes 50 1237-1243.

Larsen PJ, Enquist LW \& Card JP 1998 Characterization of the multisynaptic neuronal control of the rat pineal gland using viral transneuronal tracing. European Journal of Neuroscience 10 128-145.
Lee K, Loros JJ \& Dunlap JC 2000 Interconnected feedback loops in the neurospora circadian system. Science 289 107-110.

Menaker M 1971 Rhythms, reproduction, and photoreception. Biology of Reproduction 4 295-308.

Meyer-Bernstein EL, Jetton AE, Matsumoto SI, Markuns JF, Lehman MN \& Bittman EL 1999 Effects of suprachiasmatic transplants on circadian rhythms of neuroendocrine function in golden hamsters. Endocrinology 140 207-218.

Nagai K, Nagai N, Shimizu K, Chun S, Nakagawa H \& Niijima A 1996 SCN output drives the autonomic nervous system: with special reference to the autonomic function related to the regulation of glucose metabolism. Progress in Brain Research 111 253-272.

Palm IF, Van Der Beek EM, Wiegant VM, Buijs RM \& Kalsbeek A 1999 Vasopressin induces a luteinizing hormone surge in ovariectomized, estradiol-treated rats with lesions of the suprachiasmatic nucleus. Neuroscience 93 659-666.

Pickering TG 1990 The clinical significance of diurnal blood pressure variations. Dippers and nondippers. Circulation 81 700-702.

Plautz JD, Kaneko M, Hall JC \& Kay SA 1997 Independent photoreceptive circadian clocks throughout drosophila. Science $\mathbf{2 7 8}$ 1632-1635.

Ralph MR, Foster RG, Davis FC \& Menaker M 1990 Transplanted suprachiasmatic nucleus determines circadian period. Science $\mathbf{2 4 7}$ 975-978

Roland BL \& Sawchenko PE 1993 Local origins of some GABAergic projections to the paraventricular and supraoptic nuclei of the hypothalamus in the rat. Journal of Comparative Neurology $\mathbf{3 3 2}$ 123-143.

Romijn HJ, Sluiter AA, Pool CW, Wortel J \& Buijs RM 1997 Evidence from confocal fluorescence microscopy for a dense, reciprocal innervation between AVP-, somatostatin-, VIP/PHI-, GRP-, and VIP/PHI/GRP-immunoreactive neurons in the rat suprachiasmatic nucleus. European Journal of Neuroscience $\mathbf{9}$ 2613-2623.

Silver R, Lesauter J, Tresco PA \& Lehman MN 1996 A diffusible coupling signal from the transplanted suprachiasmatic nucleus controlling circadian locomotor rhythms. Nature 382 810-813.

Teclemariam Mesbah R, Kalsbeek A, Buijs RM \& Pøvet P 1997 Oxytocin innervation of spinal preganglionic neurons projecting to the superior cervical ganglion in the rat. Cell and Tissue Research 287 481-486.

Teclemariam Mesbah R, Ter Horst GJ, Postema F, Wortel J \& Buijs RM 1999 Anatomical demonstration of the suprachiasmatic nucleus-pineal pathway. Journal of Comparative Neurology 406 171-182.

Van der Beek EM, Horvath TL, Wiegant VM, Van Den Hurk R \& Buijs RM 1997 Evidence for a direct neuronal pathway from the suprachiasmatic nucleus to the gonadotropin-releasing hormone system: combined tracing and light and electron microscopic immunocytochemical studies. Journal of Comparative Neurology 384 569-579.

Van der Horst GT, Muijtjens M, Kobayashi K, Takano R, Kanno S, Takao M, de Wit J, Verkerk A, Eker AP, van Leenen D, Buijs R, Bootsma D, Hoeijmakers JH \& Yasui A 1999 Mammalian Cry1 and Cry2 are essential for maintenance of circadian rhythms (see comments). Nature 398 627-630.

Watts AG, Swanson LW \& Sanchez-Watts G 1987 Efferent projections of the suprachiasmatic nucleus: I. Studies using anterograde transport of Phaseolus vulgaris leucoagglutinin in the rat. Journal of Comparative Neurology 258 204-229.

Whitmore D, Foulkes NS \& Sassone-Corsi P 2001 Light acts directly on organs and cells in culture to set the vertebrate circadian clock (see comments). Nature 404 87-91.

Yamamoto M, Yamasaki Y, Kodama M, Matsuhisa M, Kishimoto M, Ozaki H, Tani A, Ueda N, Iwasaki M \& Hori M 1999 Impaired diurnal cardiac autonomic function in subjects with type 2 diabetes. Diabetes Care 22 2072-2077. 
Yamazaki S, Numano R, Abe M, Hida A, Takahashi R, Ueda M, Block GD, Sakaki Y, Menaker M \& Tei H 2000 Resetting central and peripheral circadian oscillators in transgenic rats. Science $\mathbf{2 8 8}$ 682-685.

Zhou JN, Riemersma RF, Unmehopa UA, Hoogendijk WJ, Van Heerikhuize JJ, Hofman MA \& Swaab DF 2001 Alterations in arginine vasopressin neurons in the suprachiasmatic nucleus in depression. Archives of General Psychiatry 58 655-662.

Received 3 October 2002

Accepted 19 December 2002 